\title{
A Quantitative Fractionation Method for Bilirubin in Human Bile
}

\author{
By \\ Takeo Sato and Tatsuo Saitoh \\ From the Department of Surgery, Tohoku University School of \\ Medicine, Sendai; Director: Prof. T. Maki
}

(Received for publication, August 5, 1964)

\begin{abstract}
The main ingredient of the pigmented calcium stone is calcium bilirubinate. This substance has been known to be formed by hydrolysis of bilirubin glucuronide, the commonest type of bile pigment in bile, by $\beta$-glucuronidase of bacterial origin and subsequent combination of the resulting free bilirubin with calcium. However, it also seems to be possible that a portion of bilirubin exists in bile as labile molecular compounds or conjugates which, in some instances, are able to combine with calcium to be precipitated as calcium bilirubinate. To evaluate the latter substances quantitatively, the authors established a systematic fractionation of bile pigments in bile using several solvents, and isolated some fractions consisting of labile bilirubin conjugates with phospholipids, proteins and bile acids.
\end{abstract}

It has been known that bile pigments in bile consist mostly of diglucuronide of bilirubin, so-called Pigment $\mathbf{I I}^{1}$, and include a small amount of sulfate of bilimbin ${ }^{2}$. However, a systematic method for quantitative fractionation of human bile pigments was not available until recent years. In 1942 , $\mathrm{Hara}^{3)}$ made an attempt to estimate quantitatively the content in bile of bilirubin by extractions with chloroform, but failed in establishing a satisfactory method because of the presence of an appreciable amount of mucins in bile which disturbed the fractionation procedure. Shimada ${ }^{4)}$ extracted dried canine bile with chloroform and isolated direct reacting bilirubin of the salt type and that of the ester type. He also observed that when human bile obtained by a duodenal catheterization was deproteinized with ammonium sulfate, dried and treated with chloroform, indirect reacting bilirubin and bilirubin of the salt type were well extractable, but esterified bilirubin was not.

The molecular patterns which bilirubin exhibits in bile are of practical importance with respect to the pathogenesis of calcium bilirubinate stones or socalled pigmented calcium stones. The main ingredient of this type of gallstone is calcium bilirubinate that is a compound of free bilirubin with calcium. The

$-\approx$ - 佐藤丈夫, 紊藤達雄 
combination of the latter two substances is quite easy, as shown in an in vitro experiment performed previously by Maki et al. ${ }^{5}$ ) in the authors' department. As to the major source of free bilirubin in the development of calcium bilirubinate stones, they have elucidated that when bile is infected bilirubin glucuronide is hydrolyzed by the activity of bacterial $\beta$-glucuronidase into free bilirubin and glucuronic acid. However, some other labile forms of bilirubin may also offer free carboxyl radicals and may thus participate in formation of calcium bilirubinate. In this connection, this paper is concerned with a systematic and thorough fractionation of bile pigments in bile as an avenue to the solution of the gallstone problem.

\section{EXPERIMENTAL}

I. Fractionation procedure

The scheme of the fractionation is outlined in Table I. To an aliquot of the bile sample are added 5 parts of 98 per cent ethanol and 1-3 parts of saturated ammonium sulfate solution. The mixture is allowed to stand for a while and then centrifuged. The supernatant liquid thus obtained contains all the bile pigments except protein-bound ones which are minimal on most occasions. A small amount of the liquid is taken in a test tube, mixed with methanol and subjected to the

Table I. Scheme for Fractionation of Bile Pigments in Bile

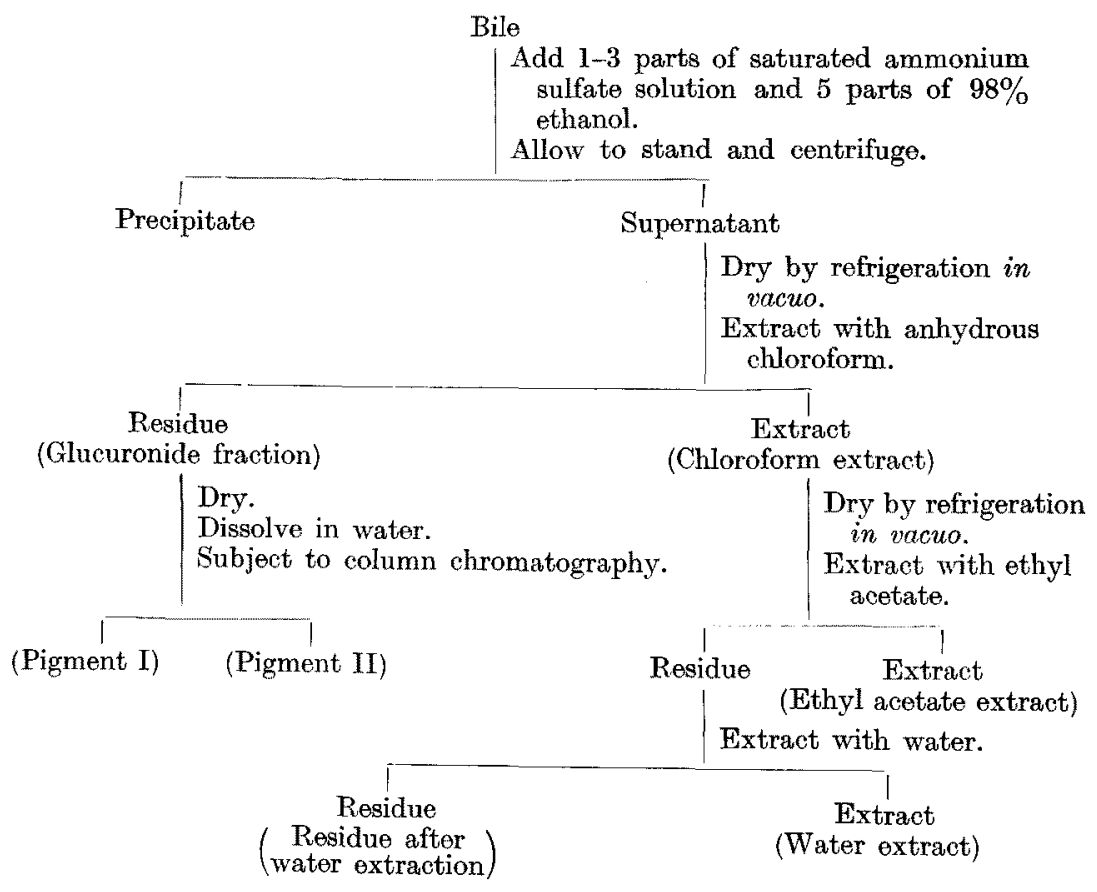


diazo reaction for quantitative estimation of total bile pigments.

The remaining portion of the supernatant is thoroughly dried by freezing in vacuo with acetone dry ice. The resulting desiccated material is then immediately mixed with chloroform taking precautions to prevent contact with air, transferred into a $\mathrm{G}_{4}$ glass filter and repeatedly extracted with anhydrous chloroform until the filtrate no longer shows the tint of bile pigments. The filtrates are pooled and measured, a portion of which is used for determination of the bile pigment content (chloroform extract).

The chloroform extract is subjected to further fractionations. For this purpose, a portion of the extract is dried by freezing in vacuo to evaporate chloroform and then extracted with ethyl acetate. The extract is mixed with methanol and the bilirubin content is estimated (ethyl acetate extract). The residue of the extraction with ethyl acetate is dried in vacuo and extracted with distilled water (water extract). The final residue is chloroform-soluble (residue after water extraction).

The bile pigments in the residue after extraction with anhydrous chloroform consist mostly of glucuronide of bilirubin or so-called esterified bilirubin. The glucuronide fraction is separated into Pigments I and II by means of a reverse phase column chromatography using a Cole and Lathe system with n-butanol at pH 6.0. The Pigment $I$ is colorimetried directly with a standard bilirubin solution and the Pigment II by the diazo reaction.

Recovery rate of the chloroform extract: In order to evaluate the recovery rate of the chloroform extract in the above procedure, a standard preparation of free dibasic acid bilirubin (Daiichi Chemical Co.) was suspended in water and put into the fractionation. In four independent experiments using $1.0 \mathrm{mg}$ each of the sample, the yield of the chloroform extract ranged from $0.904 \mathrm{mg}$ to $0.954 \mathrm{mg}$, the recovery rate being 91.8 per cent on an average (Table II). It was thus revealed that although a small portion of free bilirubin entered the precipitate in the deproteinization procedure, the major part remained in the supernatant and was

Table II. Recovery of Bilirubin in the Chloroform Extract

\begin{tabular}{c|c}
\hline Sample* & $\begin{array}{r}\text { Recovery rate } \\
(\%)\end{array}$ \\
\hline No. 1 & 95.4 \\
2 & 90.4 \\
3 & 90.4 \\
4 & 90.8 \\
\hline Average & 91.8 \\
\hline
\end{tabular}

* Each sample was prepared by suspending $1.0 \mathrm{mg}$ of free dibasic acid bilirubin in distilled water. 
recovered by the chloroform extraction following desiccation of the supernatant material. The chloroform extract was in this case absolutely water-insoluble.

II. Characteristics of each fraction from normal bile

Normal bile specimens which were obtained from the gallbladder on operation of cases without biliary disorders were fractionated by the above procedure and subjected to a one-dimension ascending paper chromatography. Paper: Toyo Filter Paper No. 2 or No. 51. Developer: chloroform, n-propanol-water (1:1) or n-butanol-ethanol-water (1:1:1). After completion of the development, the strips were stained with the diazo reagent, antimony trichloride, Sudan Black B, B.P.B., aniline hydrogen phthalate and naphthoresorcinol, and the colorized spots were identified with reference to Table III which shows the reactions of bile constituents with these reagents.

TABLE III. Colorization on Paper of Bile Constituents with Various Chromogenic Reagents

\begin{tabular}{|c|c|c|c|c|c|}
\hline Materials Reagents & $\mathrm{SbCl}_{3}$ & $\begin{array}{c}\text { Sudan } \\
\text { Black B }\end{array}$ & B.P.B. & $\begin{array}{l}\text { Naphtho- } \\
\text { resorcinol }\end{array}$ & $\begin{array}{l}\text { Aniline } \\
\text { phthalate }\end{array}$ \\
\hline Cholesterol & $+(-)$ & $\pm(-)$ & - & - & - \\
\hline Bilirubin & $+(-)$ & $+(-)$ & $-(-)$ & - & - \\
\hline Lecithin & $+(t)$ & $+(+)$ & $+(+)$ & - & - \\
\hline Palmitic acid & $-(-)$ & $+(-)$ & $+(-)$ & - & - \\
\hline Stearic acid & $+(-)$ & $+(-)$ & $+(-)$ & - & - \\
\hline Oleic acid & $+(-)$ & $+(-)$ & $+(-)$ & - & - \\
\hline Glucuronic acid & - & - & $\pm(-)$ & + & + \\
\hline Glycocholic acid & $+(t)$ & - & - & - & - \\
\hline Deoxycholic acid & $+(+)$ & $\pm(-)$ & - & - & - \\
\hline Cholic acid & $+(+)$ & $\pm(-)$ & - & - & - \\
\hline Proteins & & & + & & \\
\hline
\end{tabular}

-, no colorization; \pm , faint but perceptible colorization; + , definite coloriza.

tion. In parentheses are shown reactions after treatments of the materials with acetone for 60 minutes.

1. Chloroform extract

This fraction of bile differed from the standard bilimbin preparation (free dibasic acid bilirubin) in several aspects. For example, the former contained a pigment which was transferred into the water layer on shaking with water in a separation funnel. Moreover, the chloroform extract exhibited a positive direct diazo reaction, while the standard bilirubin was only indirectly reacting. It was thus estimated that the chloroform extract of bile contained direct reacting bilirubin besides free bilirubin if any.

As shown in Figs. 1-3 and Table IV, paper chromatographic examinations of 


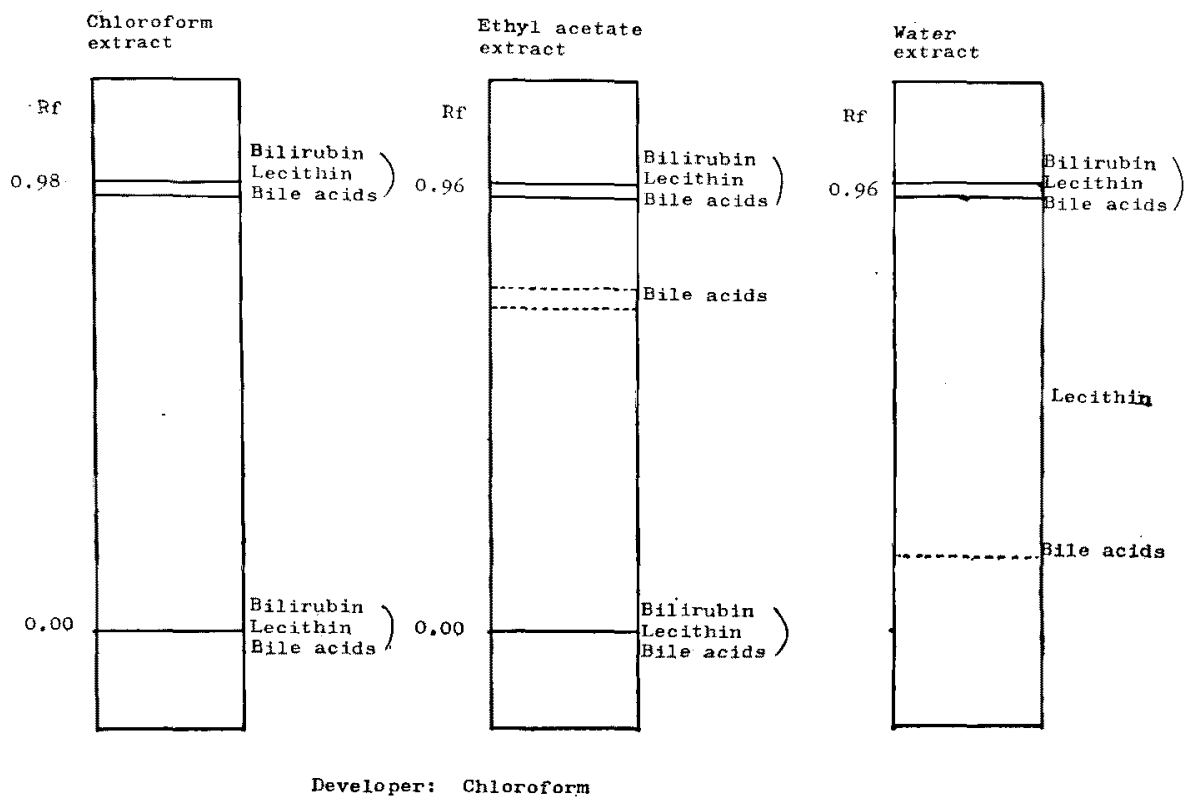

Fig. 1. Paper chromatograms of the chloroform, ethyl acetate and water extracts of bile. Solvent: chloroform.
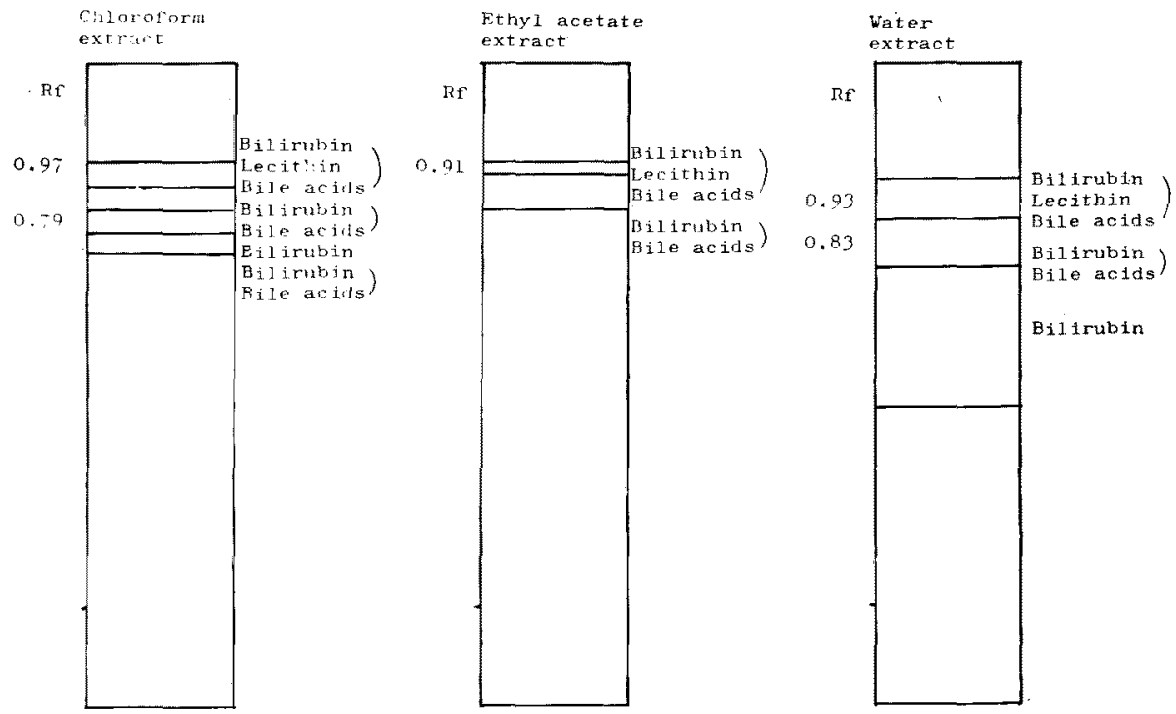

leveloper: Srnpanolmater (1:1)

Fig, 2. Paper chromatograms of the chloroform, ethyl acetate and water extracts of bile. Solvent: propanol-water (1:1). 


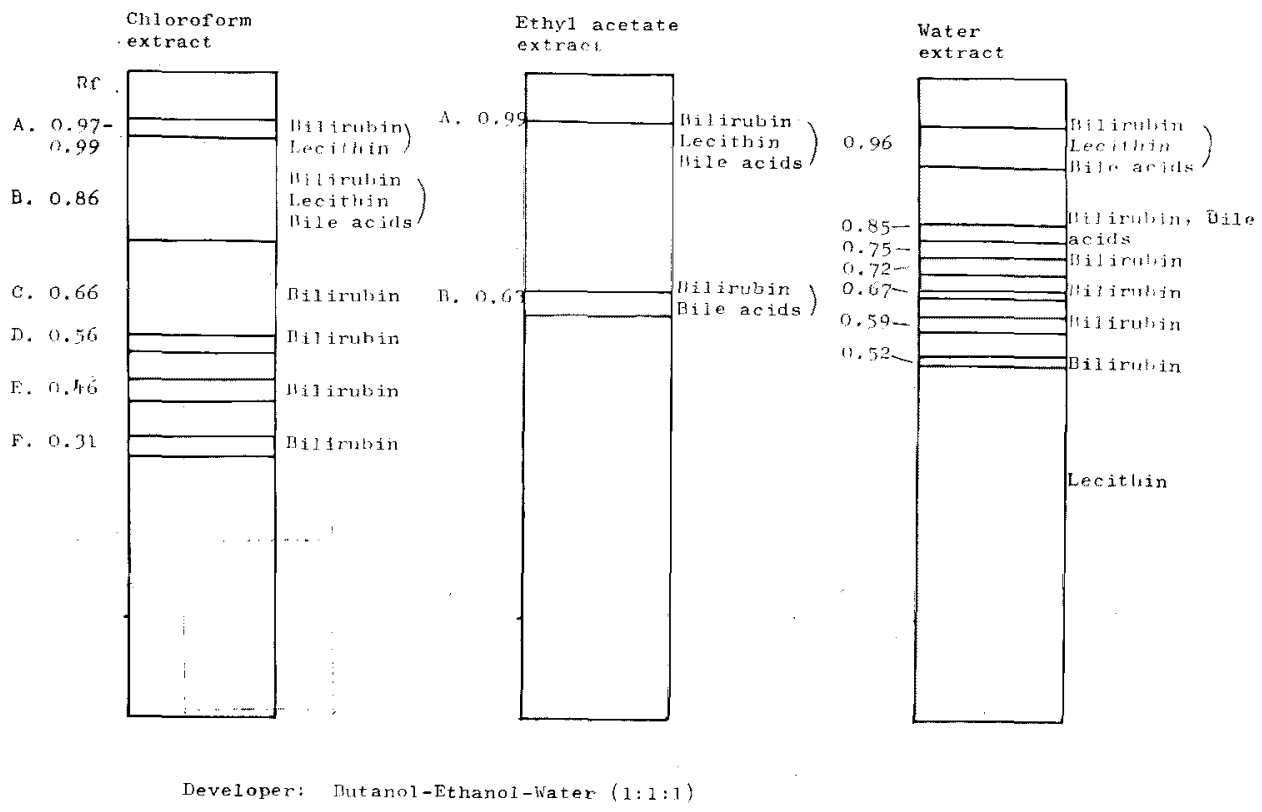

Fig. 3. Paper chromatograms of the chloroform, ethyl acetate and water extracts of bile. Solvent: butanol-ethanol-water $(1: 1: 1)$.

TABle IV. Qualitative Reactions of the Spots Obtained by Paper Chromatography of the Chloroform Extract with Butanolethanol-water $(1: 1: 1)$

\begin{tabular}{l|ccc|c|c|c|c}
\hline Spots & $\mathrm{SbCl}_{3}$ & $\begin{array}{c}\text { Sudan } \\
\text { Black B }\end{array}$ & B.P.B. & $\begin{array}{c}\text { Naphtho- } \\
\text { resorcinol }\end{array}$ & $\begin{array}{c}\text { Aniline } \\
\text { phthalate }\end{array}$ & $\begin{array}{c}\text { Diazo } \\
\text { reagent }\end{array}$ & $\begin{array}{c}\text { Possible } \\
\text { components }\end{array}$ \\
\hline A & $+(+)$ & $+(+)$ & $+(+)$ & - & - & indirect & $\begin{array}{l}\text { Phospholipids } \\
\text { Bilirubin } \\
\text { Bhospholipids }\end{array}$ \\
B & $+(+)$ & $+(+)$ & $+(+)$ & - & - & indirect? & $\begin{array}{l}\text { Phospoids } \\
\text { Bile acids } \\
\text { Bilirubin (trace) }\end{array}$ \\
C & $\pm(-)$ & $\pm(-)$ & $-(-)$ & - & - & direct & Bilirubin salts \\
D & $-(-)$ & $-(-)$ & $-(-)$ & - & - & direct & Bilirubin salts \\
E* $^{*}$ & $-(-)$ & $-(-)$ & $-(-)$ & - & - & direct & \\
F* $^{*}$ & $-(-)$ & $-(-)$ & $-(-)$ & - & - & direet & \\
$\mathbf{G}^{* *}$ & - & - & - & - & - & - &
\end{tabular}

Cf. Table III for explanation of the signs.

* Inconstant spots which may appear in normal bile specimens. **A fraction which is absent in normal bile but occasionally appears in pathologic bile specimens. This fraction does not move on ehromatography and contains no bile pigment. 
this fraction suggested that bilirubin in this fraction was conjugated with phospholipids or bile acids. However, the presence of bilirubin glucuronide was not evidenced by the chromatography. Furthermore, this fraction did not liberate glucuronic acid when incubated with $\beta$-glucuronidase.

2. Ethyl acetate extract

This fraction was soluble in ethanol, ether, acetone and chloroform but insoluble in water. The fraction indirectly reacted with the diazo reagent. Paper chromatography revealed that the pigment of this fraction was associated with phospholipids and bile acids (Figs. 1-3).

\section{Water extract}

This fraction was direct reacting with the diazo reagent and insoluble in ethyl acetate or ether. Although it was chloroform-soluble when thoroughly dried, the pigment was not extracted with chloroform from a water solution. When the water-chloroform system was slightly acidified with hydrochloric acid, the pigment was transferred into the chloroform layer. The resulting chloroform extract was indirect reacting with the diazo reagent and was extractable with water only in an alkaline reaction. On paper chromatography, the pigment was found to be associated with phospholipids (Figs. 1-3).

This fraction thus differed from the standard bilirubin preparation in various characteristics. Inasmuch as it is definitely influenced by the hydrogen ion concentration, the conjugation of this pigment seems to be of ionic type. However, the

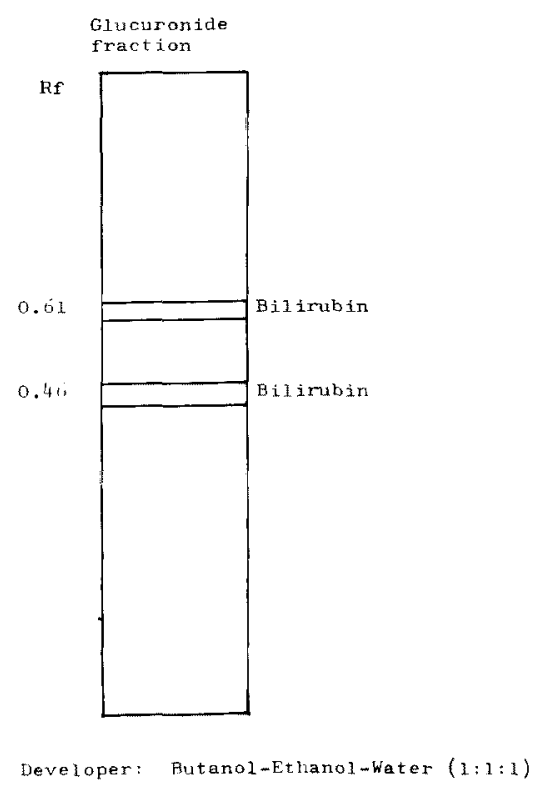

Fig. 4. Paper chromatogram of the glueuronide fraction of bile. Solvent: butanolethanol-water $(1: 1: 1)$. 
combination of phospholipids is also apparent. The pigment may be one of the forms of bilirubin which are seen only in natural bile.

4. Residue after water extraction

Normal bile is usually void of this fraction, which sometimes appears in infected bile. The pigment of this fraction is presumed to be almost identical with free dibasic acid bilirubin.

5. Glucuronide fraction

The fraction, which was obtained as the residue after chloroform extraction of the deproteinized, dried bile, consisted of glucuronides of bilirubin and was soluble in water and insoluble in chloroform, ethyl acetate, ether and acetone. On paper chromatography, the fraction exhibited two separate spots as shown in Fig. 4.

In the case of normal bile, the deposit of proteins produced by the deproteinization procedure is slightly contaminated with a yellow pigment. The pigment is soluble in water but in none of ethyl acetate, ether and chloroform, and gives a direct positive reaction with the diazo reagent. This pigment is supposed to be protein-bound bilirubin which may exist in bile in a small amount.

\section{DISCUSSION}

By means of a reverse phase column chromatography, Cole, Lathe and Billing (1954) ${ }^{1}$ ) isolated from the jaundiced human serum three different bile pigments: indirect reacting bilirubin and direct reacting Pigments $I$ and II. Subsequently, it was elucidated by Schmid ${ }^{7)}$, Billing and Lathe ${ }^{8)}$, and Talafant ${ }^{9}$ that so-called direct reacting bilirubin in serum, urine and bile was conjugates of bilirubin with glucuronic acid. These observations have stimulated further investigations on the metabolism of bile pigments.

The present study was also concerned with the quantitative fractionation of bile pigments in bile but its aim was to investigate, in relation to the pathogenesis of gallstones, if there were such forms of bilirubin that easily combine with calcium or with other inorganic elements to form deposits of insoluble salts. Therefore, both direct reacting bilirubin and indirect reacting one were put into systematic fractionations.

It is a well known fact that free bilirubin or so-called indirect reacting bilirubin is extractable with chloroform, in other words, a bile pigment which is well soluble in chloroform is mostly indirect reacting bilirubin. However, the chloroform extract prepared from the deproteinized bile specimen was found to contain not only indirect reacting bilimbin but also a perceptible amount of direct reacting bilirubin. Qualitative analyses revealed that the latter fraction included pigments combined with phospholipids or with bile acids but was void of bilirubin glucuronide.

The presence in bile of such bilirubin conjugates with substances other than glucuronic acid has been indicated in a number of previous reports. Shimada ${ }^{4}$ ) 
observed a fraction which moved on paper chromatography with lecithin and a fatty acid. Talafant ${ }^{10}$ ) suggested the presence of a conjugate of bilirubin with deoxycholic acid, and Shimura' ${ }^{11)}$ obtained by electrophoresis a bilirubin fraction with positive qualitative reactions for amino acids, bile acids and proteins. In a fractionation experiment of fatty acid in bile, Nakayama and Johnston ${ }^{12}$ ) found that a fraction which was extracted with ether-methanol (1:4) consisted of lecithin and bilirubin. Katsuki et $a l .^{13}$ ) analyzed paper-electrophoretically bile pigments of bile and suggested that bile pigments might probably be combined with substances such as bile proteins, lecithin-bile acid complex, mucoprotein or lipoprotein.

Bilirubin is water-solubilized before it is excreted from the liver. As mentioned before, the chemical process associated with this phenomenon is known to be mainly esterification and in part salt formation of bilirubin. However, the above experimental results indicate the presence of another pathway in which bilirubin is solubilized in water by conjugating with other substances such as proteins, phospholipids and bile acids. While esterification or salt formation of bilimbin involve its carboxyl group, conjugation of the above substances presumably take place at a nitrogen atom of bilirubin. It is thus very likely that the resulting molecular compounds are very labile and easily combine with calcium and other inorganic substances to form water-insoluble precipitates in bile.

When the chloroform extract was dried and treated with ethyl acetate, a pigment which was bound with phospholipids was extracted by the latter solvent. When the ethyl acetate extract was further extracted with water, the resulting extract was found to contain bilirubin fractions conjugated with phospholipids and bile acids, and also non-conjugated bilirubin of the salt type. Bilirubin-sulfate complex, whose existence in bile was claimed by Isselbacher and $\mathrm{McCarthy}^{2}$, was not identified in the present fractionation because the study did not include a radio-isotope technique.

On the other hand, the residue after chloroform extraction of the dried, deproteinized bile material contained little or no bilirubin of the salt type. They are so-called Pigments I and II and are known to represent monoglucuronide and diglucuronide, respectively, of bilirubin. The present fractionation procedure was set up to include the separation of the above two fractions since the quantitative relation between these pigments was estimated to be a matter of considerable variation according to various pathologic conditions.

Thanks are due to the Ministry of Education and the Tkyo Biochemical Institute for the grant from Soientific Research Fund to this study. T. Maki.

\section{References}

1) Cole, P.G., Lathe, G.H. \& Billing, B.H., Biochem. J., 1954, 57, 514.

2) Isselbacher, K.J. \& MoCarthy, E.A., J. clin. Invest., 1959, 38, 645. 
3) Hara, Y., Igaku Kenkyu (Jap.), 1952, 22, 70.

4) Shimada, Y., ibid., 1954, 24, 94.

5) Maki, T., Sato, Ta. \& Suzuki, N., T'ohoku J. exp. Med., 1964, 82, 117.

6) Maki, T., Sato, To. \& Sato, Ta., ibid., 1962, 77, 179.

7) Schmid, R., Science, 1956, 124, 76.

8) Billing, B.H. \& Lathe, G.H., Biochem. J., 1956, 63, 6P.

9) Talafant, E., Nature, 1956, 178, 312.

10) Talafant, E., Chem. Listy, 1954, 48, 752.

11) Shimura, H., Hirosaki Igaku (Jap.), 1957, 8, 490.

12) Nakayama, F. \& Johnston, C.G., J. Lab. clin. Med., 1962, 59, 364.

13) Katsuki, T., Shimura, H. \& Johnston, C.G., Acta med. Univ. Kagoshima, 1963, 5, 119. 